\title{
Micro-buckling of carbon fibers in shape memory polymer composites under bending in the glass transition temperature region
}

https://doi.org/10.1515/cls-2021-0009

Received Dec 28, 2020; accepted Feb 06, 2021

\begin{abstract}
The influence of a wide temperature range in the glass transition region of a shape memory polymer (SMP) matrix on micro-buckling of the fiber reinforcements in shape memory polymer composites (SMPC) under large bending deformation is described. Analytical expressions to estimate the strain energy, neutral strain surface, critical buckling surface and half wavelength of the buckled fibers in the SMPC are presented based on the minimum energy method. This study considers the reinforced fibers as three-dimensional elastic bodies and the matrix as a temperature stimulated flat plate. A comprehensive study was performed to understand the dynamic temperature behavior of the micro-buckled fibers and corresponding results were validated by previous works in the literature. The effects of fiber volume fraction and thickness of the SMPC plates on the half wavelength are also discussed along with the simultaneous influence of temperature on the parameters computed in the minimum energy analysis.
\end{abstract}

Keywords: fiber micro-buckling, shape memory polymer composites, minimum energy method, fiber filled composites, bending

\section{Introduction}

Shape memory polymers (SMP) are a category of materials sensitive to stimuli of either heat, electricity, magnetism, moisture or their combinations that regain their original shape after deformation [1-3]. SMPs have the salient features of improved shape retention, recovery strain, resis-

\footnotetext{
${ }^{\star}$ Corresponding Author: Nilesh Tiwari: Mechanical Engineering Department, Sardar Vallabhbhai National Institute of Technology, Surat, 395007, India; Email: nileshbwtiwari@gmail.com

AbdulHafiz A. Shaikh: Mechanical Engineering Department, Sardar Vallabhbhai National Institute of Technology, Surat, 395007, India; Email: aas.svr1@gmail.com
}

tance to harsh environments and low weight [4-6]. Due to these noticeable qualities, SMPs are widely used in the aviation, space technology, biotechnology, electronics and textile industries [7-11]. Moreover, incorporation of the reinforcements in the shape memory polymer composites (SMPC) can significantly enhance the mechanical properties of SMP without adversely affecting the shape memory property and recovery strain of the matrix $[12,13]$.

SMPC structures utilize the softness of the SMP matrix above its glass transition temperature $\left(T_{g}\right)$ to permit high flexural or tensile deformation devoid of permanent plasticity or failure. The deformation can be maintained even after cooling of the deformed structure and removing the external loads with the sample subsequently regaining its original structure when heated above $T_{g}[6,14]$. The characteristics of high flexural deformation of SMPC material without permanent distortion is because of the micro-buckling of the fibers in the soft SMP matrix at elevated temperature without fiber rupture, with the SMPC and fibers regaining their original shape when heated. This is in contrast with the general assumption of fiber buckling and kinking as a potential reason for polymer composite failure [15-17]. The phenomenon of micro-buckling of fibers in the SMP matrix is useful in a diverse range of applications, such as flexible sensors and actuators in electronics, deployable structures in aerospace and microtubes in living tissues [18-20].

The behavior of fiber buckling in composites, to our knowledge, was first studied by Rosen [21], in which he presented two-dimensional buckling patterns of the sinusoidal extension mode in composites with low fibers volume fraction and the sinusoidal shearing mode in the case of high volume fraction. Fiber buckling, as one way of instability, has been studied at various magnifications. Rudykh and deBotton [22] investigated the macroscopic fiber deformation in composites under high bending force, followed by similar studies under finite strain by Agoras et al. [23] and Michel et al. [24]. Slesarenko and Rudykh [25] performed the transitional study, relating microscopic and macroscopic studies in fiber composites. Guynn et al. [26, 27] performed the parametric study of micro-buckling origin in compos-

๑ Open Access. (C) 2021 N. Tiwari and A. A. Shaikh, published by De Gruyter 
ite laminates which aimed to understand micro-buckling of SMPC plates in the glass transition region under finite strain through the energy method. Campbell and Maji [28] estimated the half wavelength of micro-buckling for fibers in normal polymer composites through the minimum energy method, with the results indicating a reduction of the critical stresses for the beginning of micro-buckling with the increase in fiber volume fraction. Francis et al. [29] presented the micro-buckling parameters of elastic memory composites (EMC) under bending. Lan et al. [30] performed similar work, along with an experimental interpretation of the micro-buckling of carbon fibers in EMC. Zhang et al. [31, 32] performed an analytical investigation of EMC and functionally graded plates through an energy method considering the carbon fibers as linearly elastic 3D solids.

Numerous works are available which discuss the post buckling behavior of fibers in composite plates. In these reports, the fiber is generally considered as a one dimensional beam [28-30] or two dimensional plates [33]. Moreover, all the reports focus on the micro-buckling of the fibers with the assumption of a constant temperature environment above the matrix $T_{g}$. Even in the works concentrating on the SMPC, the micro-buckling parameters are estimated primarily for temperatures above the glass transition region of the SMP matrix [29-32]. This may result in only a partial interpretation of the effect of dynamic modulus variation on the micro-buckling of the reinforcing fibers in SMPC, due to the effect of the change of temperature on the properties of the SMP in its glass transition region.

As indicated by the above review of available literature, this work is believed to be an initial attempt to study the influence of changes in temperature in the glass transition region of the SMP matrix on the micro-buckling of carbon fibers, when SMPC plates are subjected to high deflection under pure bending with the ends of the bent plates being free and the bending force applied to the center of the rectangular plates. In addition, an analytical study was performed to estimate the fiber micro-buckling parameters with the fiber assumed to be a linearly elastic, 3D solid, considering its size effect on the shear strain of the matrix. The effect of fiber volume fraction and plate thickness on the micro-buckling behavior of the SMPC under dynamic temperature variation was also considered in the current study.

The paper is organized as follows: Section 1, above, presents a brief introduction about fiber micro-buckling based on a brief literature survey. Section 2 discusses the mathematical formulation of strains and strain energy generated during the micro-buckling phenomenon in SMPC with its temperature governed material properties. Section 3 presents the expressions of critical buckling surface posi- tion, neutral surface position and half wavelength of the buckled fibers with respect to temperature. In section 4, the results of calculations of the critical buckling surface position, neutral surface position and half wavelength of the buckled fibers with respect to temperature are described and compared with the results of Zhang et al. [31] Section 5 presents the conclusions based on the findings of our study.

\section{Mathematical modelling}

Correlations are presented to calculate the micro-buckling parameters of SMPC plates under high strain bending for a wide range of temperature near $T_{g}$. To achieve this initially the basic equations governing the micro-buckling are stated which is then followed with material modelling of the temperature dependent SMP matrix and the corresponding carbon fiber reinforced SMPC. Then the properties of the SMPC are substituted into the equations to obtain the influence of dynamic temperature variations on the carbon fiber micro-buckling and the results are compared with those in previous works in the literature [30, 31].

\subsection{Formulation of strain in SMPC plates}

In this study the carbon fibers were considered to be oriented uniformly in the SMP matrix (an acrylate-based polymer) in the $x$-axis direction with the bond between the reinforcement and matrix considered to be perfect with no damage or failure of the SMPC structure in the course of bending with the force applied in the $y$ direction. The SMPC plates with length $l$, width $w$ and thickness $t$, were considered for the present analysis. During the initial phase of bending, there is no indication of micro-buckling of the fibers and the neutral surface corresponds with the mid surface layer parallel to the surface in the plate with no change in the dimensions. When the magnitude of bending reaches a particular threshold value, the fibers begin to buckle on the compression side and the neutral surface moves in the direction of the tensile side to maintain equilibrium of the deformed plate. Three zones are created in this phase: compression side with buckled fibers $\left(-t / 2, z_{b}\right)$, compression side with unbuckled fibers $\left(z_{b}, z_{n}\right)$ and tension side with unbuckled fibers $\left(z_{n}, t / 2\right)$ as indicated in Figure 1 [31]. The coordinates of the neutral strain surface and the critical buckling surface are indicated by $z_{n}$ and $z_{b}$ respectively. The views of the original and bent sample in the specimens under study are given in Figures 2(a) and 2(b), respectively. 


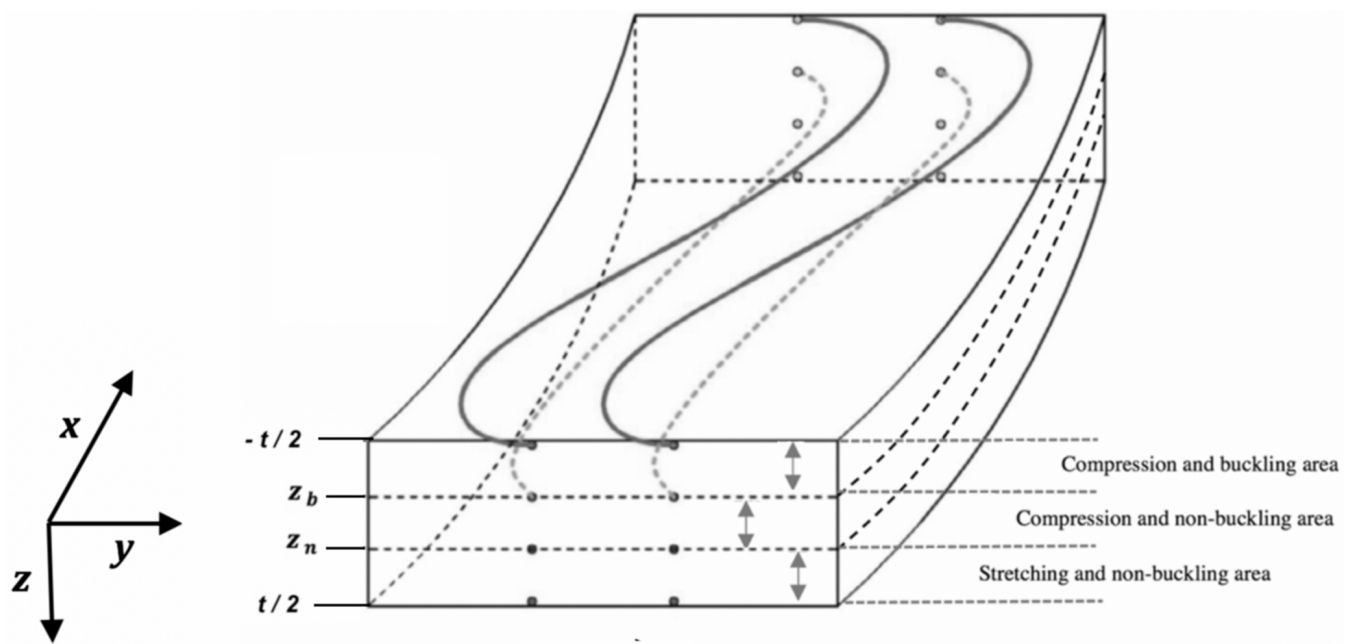

Figure 1: The buckling deformation of fibers in the SMPC plate, here $z_{n}$ and $z_{b}$ denote the neutral strain surface and the critical buckling surface respectively, with the surfaces parallel to the $x z$ surface [31]

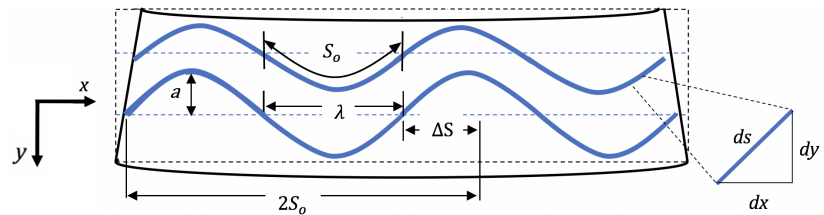

Figure 2: Magified section of the SMPC with a carbon fiber reinforcement in the SMP matrix indicating the microbuckling of the fiber in the SMPC under large strain deformation. Note: the microbuckling of fibers is greatly exagerated in the figure. The total length of the buckled fiber is the same as that of the original plate

During bending of the SMPC plate, the fiber undergoes in-plane micro-buckling, which results in a harmonic shift of the fiber in the $y$ axis direction (plate width). The resultant sinusoidal trajectory of the micro-buckled fiber is indicated as,

$$
y(x)=\operatorname{asin}\left(\frac{\pi x}{\lambda}\right)
$$

where $a$ indicates the amplitude of the sinusoidal wave of the buckled fibers and $\lambda$ is the corresponding half wavelength. The fiber is considered to be incompressible which results in identical total length $\left(2 S_{o}\right)$ pre and post buckling, depicted in Figure 2(b). The arc length is indicated by Eq. 2 through substitution of the differential arc length from the Pythagoras theorem.

$$
S_{o}=\int_{0}^{S_{o}} d s=\int_{0}^{\lambda} \sqrt{d x^{2}+d y^{2}} d x
$$

The engineering strain $\left(\varepsilon_{x x}\right)$ is calculated as

$$
\varepsilon_{x X}=\frac{\Delta S}{2 S_{o}}=1-\frac{\lambda}{S_{o}}
$$

As per Kirchhoff's Theory, the strain of the bent SMPC plate is proportional to a function of the distance from the neutral strain surface $\left(z_{n}\right)$, which behaves in linearity along the thickness.

$$
\varepsilon_{X X}=k\left(z-z_{n}\right)
$$

where $k$ is the mid-plane bending curvature (unit: $\mathrm{m}^{-1}$ ) of the SMPC plate and $\varepsilon_{x x}$ indicates the strain in the $x$ direction.

Francis presented the value of the amplitude $(a)$ of the sinusoidal buckled fiber as a function of thickness for the positive values of $\left(z_{n}-z\right)$ as [29]

$$
a(z)=\frac{2 \lambda}{\pi} \sqrt{k\left(z_{n}-z\right)}
$$

From Eqs. 1 and 5, the magnitude of displacement of micro buckled fiber in the $y$ direction is

$$
y(x, z)=\frac{2 \lambda}{\pi} \sqrt{k\left(z_{n}-z\right)} \sin \left(\frac{\pi x}{\lambda}\right)
$$

In the buckling zone of the SMPC plate, the magnitude of stretch deformation for the matrix is negligible as compared to the corresponding shear deformation. $\gamma_{x y}$ and $\gamma_{y z}$ are the shear strain of matrix due to the deflection of the fiber in the $z$ direction along the $x y$ and $y z$ plane respectively as shown in Figures 3 and 4. The similar shear component in the plane parallel to the $x z$ surface has an infinite value which is eliminated. Moreover, the stiffness of the fiber is considerably greater than that of the matrix, due to which the deflection of the matrix during buckling is neglected. In the unbuckling zone, the stretch deformation occurs in the $x$ axis direction, whereas the corresponding components along the $y$ and $z$ axis are zero. 


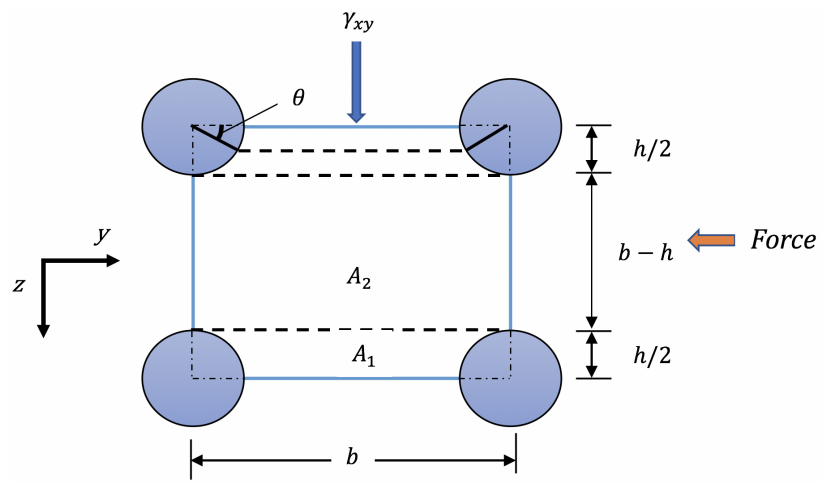

Figure 3: Distortion of the unit cell generated by strain in the $x y$ plane $\left(\gamma_{x y}\right) ; b$ is the distance between the adjacent fibers and $h$ is the diameter of the fiber

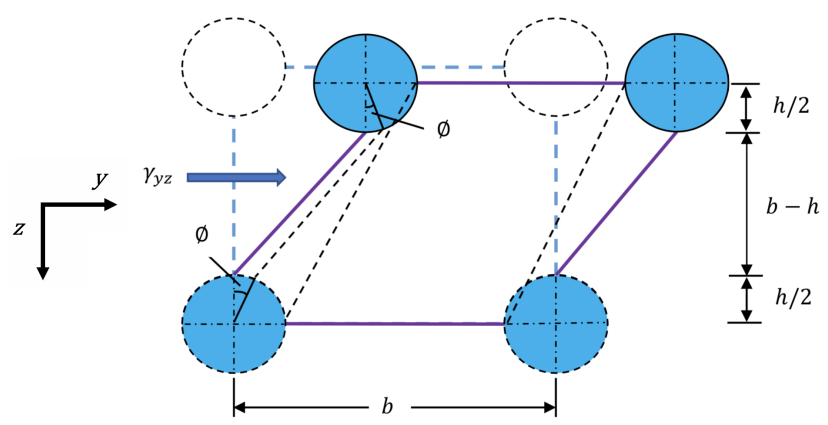

Figure 4: Distortion of the unit cell generated by strain in $y z$ plane $\left(\gamma_{y z}\right) ; b$ is the distance between the adjacent fibers and $h$ is the diameter of the fiber

The fiber is assumed to be a 3D body with consideration of its dimensions while defining $\gamma_{x y}$ and $\gamma_{y z}$. The shear strain along the $x y$ plane $\left(\gamma_{x y}\right)$ is indicated by

$$
\gamma_{x y}=\frac{\partial v}{\partial x}+\frac{\partial u}{\partial y}
$$

where $u$ and $v$ are the displacement field vectors in the $x$ and $y$ direction, respectively.

Due to the consideration of the size of the fiber, the distortion in the matrix is non-uniform when surrounding each fiber. Thus, based on the geometry indicated in Figure $5, \gamma_{x y}$ is

$$
\gamma_{x y}=\frac{\partial y}{\partial x}+\frac{\Delta u}{\Delta y}
$$

The displacement values, $\Delta u$ and $\Delta y$, are given by

$$
\begin{aligned}
& \Delta u=\frac{h \cos \theta}{2} \frac{\partial y}{\partial x} \\
& \Delta y=\frac{b-h \cos \theta}{2}
\end{aligned}
$$

where $h$ is the diameter of the fiber, $b$ is the distance between centers of two adjacent fibers, $\theta$ is the angular measurement between $0^{\circ}$ to $90^{\circ}$ with $0^{\circ}$ being measured from

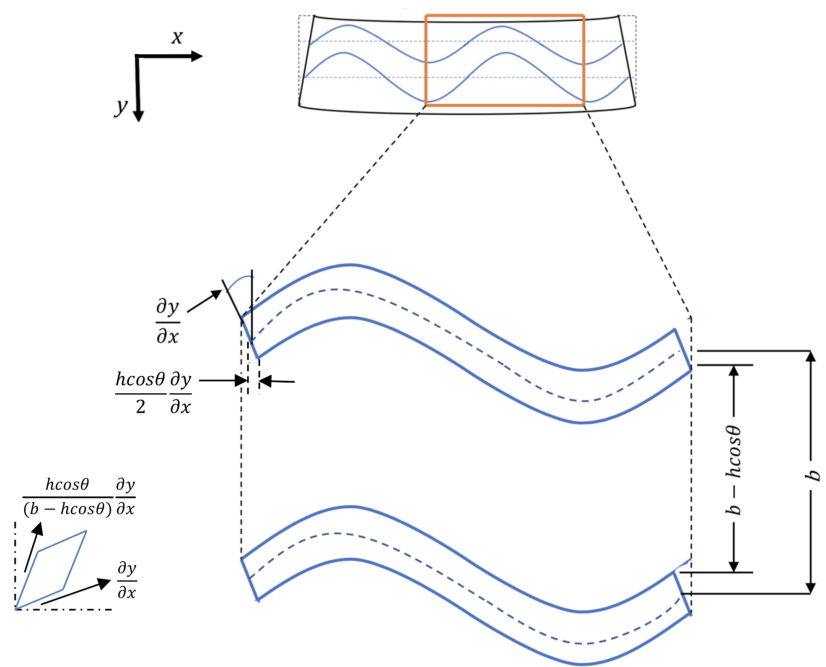

Figure 5: Deformation due to strain in the $x y$ plane $\left(\gamma_{x y}\right)$ on a magnified section [31]

the horizontal direction ( $x$ axis) and $h \cos \theta$ is the length of the horizontal projection of fiber diameter $h$ parallel to $y$ direction (Figure 5). The magnitude of $b$ is

$$
b=\sqrt{\frac{\pi h^{2}}{4 V_{f}}}
$$

where $V_{f}$ is the volume fraction of the fibers reinforcement in the SMPC plate.

Thus, the shear strain along $x y$ plane for $\theta$ between $0^{\circ}$ and $90^{\circ}$ with respect to a horizontal line parallel to the $y$ axis is,

$$
\gamma_{x y}=\frac{h \cos \theta}{b-h \cos \theta} \frac{\partial y}{\partial x}+\frac{\partial y}{\partial x}=\frac{b}{b-h \cos \theta} \frac{\partial y}{\partial x}
$$

The maximum and minimum magnitude of $\gamma_{x y}$ is at $0^{\circ}$ and $90^{\circ}$ respectively, expressed as

$$
\begin{gathered}
\gamma_{x y}^{\max }=\max \left(\gamma_{x y}\right)=\frac{b}{b-h} \frac{\partial y}{\partial x} \\
\gamma_{x y}^{\min }=\min \left(\gamma_{x y}\right)=\frac{\partial y}{\partial x}
\end{gathered}
$$

Then the shear strain of the SMP matrix in the $y z$ plane $\left(\gamma_{y z}\right)$ is

$$
\gamma_{y z}=\frac{\partial v}{\partial z}+\frac{\partial w}{\partial y}
$$

In the expression of $\gamma_{y z}$, the amplitude of sinusoidal trajectories generated during micro-buckling of the fibers is predominant at different locations in $z$ direction as shown in Figure 4, therefore $\gamma_{y z}$ is stated as

$$
\gamma_{y z}=\frac{\Delta y}{\Delta z}
$$


where the values of $\Delta y$ and $\Delta z$ are

$$
\begin{gathered}
\Delta y=\Delta a(z) \sin \left(\frac{\pi x}{\lambda}\right) \\
\Delta z=b-h \cos \varnothing
\end{gathered}
$$

where $\varnothing$ is the angular measurement of displacement of the matrix under strain between $0^{\circ}$ and $90^{\circ}$ and $h \cos \varnothing$ equals the length of the vertical projection of the fiber diameter $h$ (parallel to the $z$-axis). The value of $\Delta a$ as a function of $z$ is

$$
\Delta a(z)=a(z)-\Delta a(z-b)
$$
is

Thus, the expression for $\gamma_{y z}$ for $\varnothing$ between $0^{\circ}$ and $90^{\circ}$

$$
\gamma_{y z}=\frac{\Delta a(z)}{b-h \cos \varnothing} \sin \left(\frac{\pi x}{\lambda}\right)
$$

The maximum and minimum values of $\gamma_{y z}$ are at $\varnothing$ is equal to $0^{\circ}$ and $90^{\circ}$ respectively.

$$
\begin{gathered}
\gamma_{y z}^{\min }=\min \left(\gamma_{y z}\right)=\frac{\Delta a(z)}{b} \sin \left(\frac{\pi x}{\lambda}\right) \\
\gamma_{y z}^{\max }=\max \left(\gamma_{y z}\right)=\frac{\Delta a(z)}{b-h} \sin \left(\frac{\pi x}{\lambda}\right)
\end{gathered}
$$

The magnitude of the strain in SMPC in the $x$ coordinate for mid plane bending curvature $(k)$ is expressed as

$$
\varepsilon_{X X}=k\left(z-z_{n}\right)
$$

\subsection{Material properties of the SMPC}

The fiber reinforced SMPC considered in the current study has a dynamic modulus in the glass transition region of the SMP matrix. The properties of the SMP could be experimentally derived from dynamic mechanical analysis (DMA) or from the curve fitting of available previous DMA data. In the present study the curve fits data of the experiments conducted by Westbrook et al. [34] on an acrylate-based network polymer, were considered in all the following studies to estimate the material properties of the SMP matrix and its SMPC, followed with the study of the micro-buckling behavior of SMPC plates.

The SMP matrix was considered to be a combination of frozen and active parts, the relative amounts of which, at any temperature, were regulated by the variation of the temperature from its glass transition temperature. Based on this assumption, the temperature dependent modulus of the matrix was presented by Gu et al. [35] as

$$
E_{m}(T)=\left(E_{1}-E_{2}\right) \cdot \exp \left(-\left(\frac{T}{T_{\beta}}\right)^{m_{1}}\right)
$$

$$
\begin{aligned}
& +\left(E_{2}-E_{3}\right) \cdot \exp \left(-\left(\frac{T}{T_{g}}\right)^{m_{2}}\right) \\
& +E_{3} \cdot \exp \left(-\left(\frac{T}{T_{f}}\right)^{m_{3}}\right)
\end{aligned}
$$

where $E_{1}, E_{2}$ and $E_{3}$ are the values of storage moduli at the beginning of the glass transition, at $T_{g}$ and in the flow region at the end of the glass transition region and $T_{\beta}, T_{g}$ and $T_{f}$ are the corresponding temperatures. $m_{1}, m_{2}$ and $m_{3}$ are the Weibull parameters of optimized curve fitting. The storage modulus was assumed to be the same as the elastic modulus of the considered matrix as the loss modulus was negligible as compared to the storage modulus [35].

The temperature dependent shear modulus $\left(G_{m}(T)\right)$ and Poissons ratio $\left(\mu_{m}(T)\right)$ were given by Qi et al. [36] as

$$
\begin{gathered}
G_{m}(T)=\frac{E_{m}(T)}{2\left[1+\mu_{m}(T)\right]} \\
\mu_{m}(T)=\mu_{g} v_{g}(T)+\mu_{r}\left[1-v_{g}(T)\right]
\end{gathered}
$$

where $\mu_{g}$ and $\mu_{r}$ are Poisson's ratio of the frozen and active phases, respectively, and $v_{g}$ is the volume fraction of the frozen phase. The values of $v_{g}(T)$ were calculated as

$$
v_{g}(T)=1-\frac{1}{1+\exp \left[-\left(T-T_{m}\right) / Z\right]}
$$

where $T_{m}$ is the reference temperature and $Z$ is a parameter characterizing the width of the glass transition zone.

The elastic modulus of SMPC $\left(E_{t}\right)$ with the reinforcement of the fibers in the SMP matrix is assumed to be given by the Rule of Mixtures as

$$
E_{t}(T)=E_{f} V_{f}+E_{m}(T) V_{m}
$$

where $V_{f}$ and $V_{m}$ are the volume fractions of fiber and matrix and $E_{f}$ is the elastic modulus of fibers. Few thermomechanical studies had been performed based on finite element methods [37-41], however in the present study focus was to present the analysis based on simplified model.

The parameters calculated in Eqs. (25) and (28) were utilized in the estimation of the shear and longitudinal stress based governing equations, stated as

$$
\begin{aligned}
& \tau_{x y}=G_{m}(T) \gamma_{x y} \\
& \tau_{y z}=G_{m}(T) \gamma_{y z} \\
& \sigma_{x x}=E_{t}(T) \varepsilon_{x x}
\end{aligned}
$$




\subsection{Formulation of the strain energy in the SMPC plates}

The cumulative strain energy of the SMPC plate $(U)$ has two components: temperature dependent and temperature independent. The temperature dependent component includes three parts: shear strain energy of the matrix along the $y z$ plane $\left(U_{y z}\right)$, shear strain energy of the matrix along the $x y$ plane $\left(U_{x y}\right)$ and stretch strain energy of the SMPC $\left(U_{s t r}\right)$. The strain energy of the fiber $\left(U_{f}\right)$ was the only temperature independent component considered in our analysis of the micro-buckling.

$$
U=\underbrace{U_{y z}+U_{x y}+U_{s t r}}_{\text {Temperature Dependent }}+\overbrace{U_{f}}^{\text {Temperature Independent }}
$$

\subsubsection{Shear strain energy in the xy plane, $U_{x y}$}

The shear strain energy of the SMP matrix along the $x y$ plane in area $A_{1}$ and its symmetrical side of the unit cell indicated in Figure 3 is expressed as

$$
u_{x y}=\tau_{x y} \gamma_{x y} A_{1}
$$

where $A_{1}=\int_{0}^{\frac{\pi}{2}}\left[\frac{h}{2}(b-h \cos \theta) \cos \theta\right] d \theta$

Corresponding strain energy in the remaining area $\left(A_{2}\right)$ of the unit cell is given as

$$
\tilde{u}_{x y}=\frac{1}{2} \tau_{x y}^{\min } \gamma_{x y}^{\min } A_{2}
$$

where, $A_{2}=b(b-h)$

The shear strain energy density $\left(\tilde{U}_{x y}\right)$ of the SMP matrix in the surface parallel to the $x y$ plane is given by

$$
\tilde{U}_{x y}=\frac{u_{x y}+\tilde{u}_{x y}}{b^{2}}
$$

The total shear strain of the SMP along the whole buckling zone of the composite is given by

$$
U_{x y}=\iiint \tilde{U}_{x y} d V
$$

The expansion of Eq. (36) results in the representation of the expression as

$$
U_{x y}\left(T, z_{n}, z_{b}\right)=G_{m}(T) k w l f\left(V_{f}\right) g\left(z_{n}, z_{b}\right)
$$

where $G_{m}(T)$ is the temperature regulated shear modulus of the SMP matrix, $k$ is the mid plane bending curvature, $w$ is the width of the SMPC plate, $l$ is the length of the SMPC plate, $z_{n}$ is the distance to the neutral strain surface from the upper surface, $z_{b}$ is the distance the critical buckling surface from the upper surface and the related microbuckling geometry dependent factors, $f\left(V_{f}\right)$ and $g\left(z_{n}, z_{b}\right)$ are given by [31]

$$
\begin{aligned}
f\left(V_{f}\right) & =1-\frac{\pi}{2}-\sqrt{\frac{4 V_{f}}{\pi}} \\
& +\frac{2 \sqrt{\pi}}{\sqrt{\pi-4 V_{f}}} \arctan \left(\frac{\sqrt{\pi-4 V_{f}}}{\sqrt{\pi}-\sqrt{4 V_{f}}}\right) \\
& g\left(z_{n}, z_{b}\right)=\left(z_{n} z_{b}+\frac{z_{n} t}{2}-\frac{z_{b}^{2}}{2}+\frac{t^{2}}{2}\right)
\end{aligned}
$$

\subsubsection{Shear strain energy in the yz plane, $U_{y z}$}

Based on an approach similar to that used to estimate $U_{x y}$, the temperature regulated shear strain energy in the $y z$ plane $\left(U_{y z}\right)$ of the SMP matrix in the buckling zone is given as

$$
U_{y z}\left(T, \lambda, z_{n}, z_{b}\right)=\frac{G_{m}(T) k w l \lambda^{2}}{\pi^{2}} f\left(V_{f}\right) f\left(z_{n}, z_{b}\right)
$$

where $\lambda$ is the half wavelength of the micro buckled fiber in the $x y$ plane and $f\left(z_{n}, z_{b}\right)$ is the geometric factor with the approximation that the fiber spacing, $b$, is negligible as compared to the total thickness, $t$, stated as

$$
\begin{aligned}
& f\left(z_{n}, z_{b}\right)=\frac{1}{4 b^{2}}\left[-\left(2 z_{n}-2 z_{b}+b\right)^{2}\right. \\
& +2\left(2 z_{n}-2 z_{b}+b\right) \sqrt{\left(z_{n}-z_{b}\right)\left(z_{n}-z_{b}+b\right)} \\
& \left.+b^{2} \ln \left(\frac{2\left(t+2 z_{n}+b\right)}{2 \sqrt{\left(z_{n}-z_{b}\right)\left(z_{n}-z_{b}+b\right)}\left(2 z_{n}-2 z_{b}+b\right)}\right)\right]
\end{aligned}
$$

\subsubsection{Stretch strain energy of the SMPC, $U_{\text {str }}$}

The strain energy in the SMPC plate due to stretching in the area below $z_{n}$ within the glass transition region of the considered SMP is expressed as

$$
U_{s t r}=\frac{1}{2} \iiint \sigma_{x x} \varepsilon_{x x} d V
$$

Integration of this expression results in

$$
U_{s t r}\left(T, z_{n}, z_{b}\right)=\frac{E_{T}(T) w l}{24} k^{2} H\left(z_{n}, z_{b}\right)
$$

where $H\left(z_{n}, z_{b}\right)=\left(t^{3}-6 t^{2} z_{n}+12 t z_{n}^{2}+24 z_{n} z_{b}^{2}-24 z_{n}^{2} z_{b}-\right.$ $\left.8 z_{b}^{3}\right)$ 


\subsubsection{Strain energy of the fibers, $U_{f}$}

When the SMPC plates are subjected to bending, the fiber reinforcement in the SMP matrix undergoes micro-buckling through the whole thickness of the plate, which generates strain in the fiber. The strain energy in a single buckled fiber is expressed as

$$
U_{f, s}=\frac{E_{f} I_{f}}{2} \int_{0}^{l}\left(\frac{\partial^{2} y}{\partial^{2} x}\right)^{2} d x
$$

where $E_{f}$ and $I_{f}$ are longitudinal modulus and inertia of the fiber.

The strain energy density of the buckled fiber, based on the RVE model, is given as

$$
\bar{U}_{f}=\frac{U_{f, s}}{b^{2} l}
$$

Based on the integration of Eq. (45), the strain energy in the fiber over the entire volume $(V)$ is stated as

$$
U_{f}=\iiint \bar{U}_{f} d V
$$

The solution of expression in Eq. (46), results in

$$
U_{f}\left(\lambda, z_{n}, z_{b}\right)=\frac{4 E_{f} I_{f} V_{f} \pi k w l}{\lambda^{2} h^{2}} g\left(z_{n}, z_{b}\right)
$$

\section{Micro-buckling parameters of SMPC}

The location of the neutral strain surface $\left(z_{n}\right)$, the critical buckling surface $\left(z_{b}\right)$ and the half wavelength of the fiber $(\lambda)$ under buckling due to bending of the SMPC plates are estimated below with respect to temperature variations in the glass transition region of the SMP matrix.

\subsection{Estimation of $z_{n}$ and $z_{b}$}

The positions of $z_{n}$ and $z_{b}$ were evaluated through minimization of the total strain energy following the minimum energy method. To obtain the results in closed form, $U_{y z}$ and $U_{f}$ terms were neglected in Eq. (32), which then gives the total strain energy $\left(U_{z}\right)$ along width at any $x y$ plane as

$$
U_{z}\left(T, z_{n}, z_{b}\right)=U_{x y}+U_{s t r}
$$

The minimization of Eq. (48) with respect to $z_{n}$ and $z_{b}$ was performed by assuming $\frac{\partial U_{z}}{\partial z_{n}}=0$ and $\frac{\partial U_{z}}{\partial z_{b}}=0$. The minimum energy method presents the solutions for $z_{n}$ and $z_{b}$ as

$$
\begin{aligned}
& z_{n}=\frac{t}{2}-\frac{1}{2 k} \frac{G_{m}(T) f\left(V_{f}\right)}{E_{t}(T)} \sqrt{1+\frac{4 k t E_{t}(T)}{G_{m}(T) f\left(V_{f}\right)}}-1 \\
& z_{b}=\frac{t}{2}-\frac{1}{2 k} \frac{G_{m}(T) f\left(V_{f}\right)}{E_{t}(T)} \sqrt{1+\frac{4 k t E_{t}(T)}{G_{m}(T) f\left(V_{f}\right)}}+1
\end{aligned}
$$

The values of $z_{b}$ are thus equal to $z_{n}-\frac{1}{k} \frac{G_{m}(T) f\left(V_{f}\right)}{E_{t}(T)}$

\subsection{Estimation of the half wavelength for the fibers $(\lambda)$}

There are terms in the cumulative strain energy which deals with the half wavelength of fiber $(\lambda): U_{y z}$ and $U_{f}$. Thus, to apply the minimum energy method, these terms were minimized with respect to $\lambda$.

$$
\frac{\partial U_{\lambda}}{\partial \lambda}=\frac{\partial}{\partial \lambda}\left[U_{f}\left(\lambda, z_{n}, z_{b}\right)+U_{y z}\left(T, \lambda, z_{n}, z_{b}\right)\right]=0
$$

The solution of Eq. (51) presents a complex expression, whereas through the assumptions considered in Ref. [31], it was noticed that the variation of the mid plane bending curvature $(k)$ does not affect the half wavelength but it is regulated by the thickness $(t)$ of SMPC. Therefore, to simplify the numerical solution, for an infinite value of $k$ for $z_{n}=z_{b}=t / 2$ was assumed. Hence the simplified solution of $\lambda$ across the glass transition temperature region of the SMPC can be stated as

$$
\lambda=\left[\frac{E_{f} V_{f} \pi^{4} h^{2} t^{2}}{8 G_{m}(T) f\left(V_{f}\right)\left\{-1+\ln \left(\frac{8 t}{h} \sqrt{\frac{V_{f}}{\pi}}\right)\right\}}\right]^{\frac{1}{4}}
$$

\section{Results and discussion}

The results of the calculations of the mechanical properties of the SMPC, strain energy, position of $z_{n}$ and $z_{b}$ and half wavelength $(\lambda)$ of the buckled fibers under temperature variation in the glass transition region of the SMP matrix are presented in this section. A parametric study was also performed to understand the effects of temperature and $k$ on the total strain energy and $z_{n}$ and $z_{b}$ and the effects of the variation of temperature, thickness $(t)$ and volume fraction $\left(V_{f}\right)$ on $\lambda$. A MATLAB program was developed by us to evaluate the mathematical formulations, which were verified in subsequent stages of the work based on published results. 
Table 1: Values of the parameters used to model the SMP matrix [35]

\begin{tabular}{|c|c|c|c|c|c|c|c|c|c|c|c|}
\hline Parameter & $T_{g}$ & $T_{m}$ & $T_{\beta}$ & $T_{f}$ & $E_{1}$ & $E_{2}$ & $E_{3}$ & $m_{1}, m_{2}, m_{3}$ & $\mu_{g}$ & $\mu_{r}$ & $Z$ \\
\hline Values & 305 & 300.5 & 295.2 & 415.5 & 2.552 & 1.876 & 5 & $19.3,58.4,177.6$ & 0.35 & 0.49 & 7 \\
\hline Unit & $K$ & $K$ & $K$ & $K$ & $G P a$ & $G P a$ & $G P a$ & - & - & - & - \\
\hline
\end{tabular}

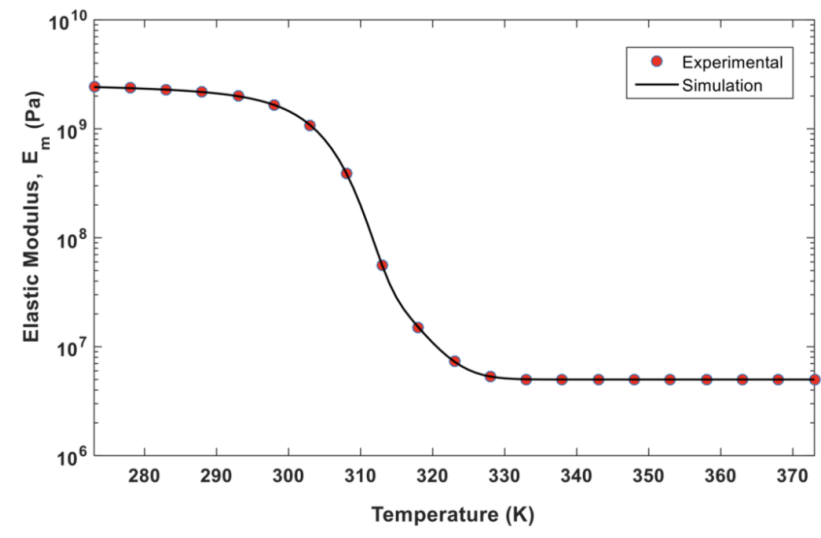

(a)

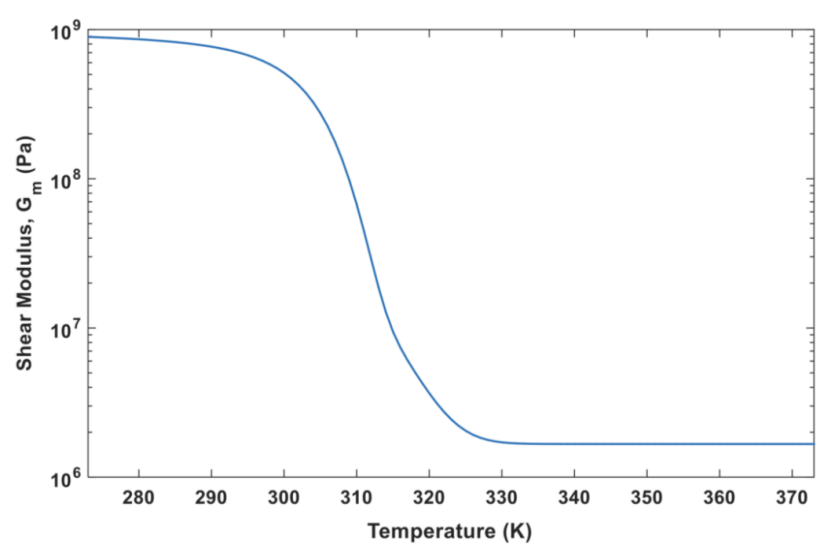

(b)

Figure 6: Modulus of SMP in glass transition region: (a) elastic modulus; (b) shear modulus

\subsection{Temperature regulated properties of SMPC}

The elastic $\left(E_{m}(T)\right)$ and shear moduli $G_{m}(T)$ of the SMP matrix with respect to temperature are depicted in Figures 6(a) and 6(b) based on Eq. (24) to (27). The parameters substituted in these equations are summarized in Table 1 and were based on the analytical investigations performed by Gu et al. [35] The present predictions of $E_{m}(T)$ were in appreciable congruence with their results, which validates the calculations of the current work. The trajectories in Figures 6(a) and 6(b) highlight the dynamic material prop- erties of the SMP matrix in the glass transition region. In the initial stage of temperature elevation, the elastic and shear moduli remained constant, representing the elastic nature of the material properties, but during the transition from the beginning of the glass transition temperatures to the flowing temperature the modulus drastically declined and then stabilized to remain constant after the transition. The drastic change in the material properties significantly governs the bending of the corresponding composites which is elaborated subsequently to understand the effect of temperature rise on micro-buckling of fibers under bending.

The elastic modulus of the SMPC in the glass transition temperature region was calculated by substitution of the properties of the SMP matrix in Eq. (28) along with the characteristics of the carbon fiber reinforcement. The longitudinal modulus of the carbon fiber was considered to be $276 \mathrm{GPa}[30]$ and its volume fraction was taken as 0.4 for the purpose of validation of the current work. The temperature governed properties of the SMPC were utilized in the following stages to estimate the strain energy in the SMPC plates, the micro-buckling parameters of the fibers and the SMPC strain surface positions.

\subsection{Strain energy}

The total strain energy of the SMPC plate under bending includes the strain energy of the SMP in the $x y$ plane $\left(U_{x y}\right)$, the strain energy of the SMP in the $y z$ plane $\left(U_{y z}\right)$, the strain energy in the SMPC $\left(U_{s t r}\right)$, and the strain energy in the fibers $\left(U_{f}\right)$. The formulations of these strain energies were presented in section 2.3. Distinct strain energies were calculated by Eqs. (37), (40), (43) and (47) and substituted in Eq. (32) to evaluate the cumulative strain energy $(U)$. Figure 7 indicates the behavior of $U$ with respect to the $z_{n} / t$ ratio for the various values of $k, 5,10$ and $20 \mathrm{~m}^{-1}$, with the material properties of the fibers and the geometry of the SMPC summarized in Table 2. The results were in close proximity to those in Ref. [31]. It is also noticed that the values of $U$ were approximately the same as the sum of $U_{x y}$ and $U_{s t r}$, highlighting the negligible influence of $U_{y z}$ and $U_{f}$ on the strain energy generated during the bending of the SMPC plates. 
Table 2: Values of the parameters used to model the micro-buckling of the fibers in the SMPC at elevated temperature [31]

\begin{tabular}{c|ccccccc}
\hline Parameter & $\begin{array}{c}\text { Fiber } \\
\text { diameter } \\
h\end{array}$ & $\begin{array}{c}\text { Fiber } \\
\text { volume } \\
\text { fraction }\end{array}$ & $\begin{array}{c}\text { Plate } \\
\text { Thickness } \\
t\end{array}$ & $\begin{array}{c}\text { Plate } \\
\text { Width }\end{array}$ & $\begin{array}{c}\text { Plate } \\
\text { Length }\end{array}$ & $\begin{array}{c}\text { Longitudinal modulus } \\
\text { of the fibers } \\
l\end{array}$ & $\begin{array}{c}\text { Half } \\
\text { wavelength } \\
\text { Values }\end{array}$ \\
$\begin{array}{c}\text { Unit } \\
7 \times 10^{-3}\end{array}$ & 0.2 & 2 & 5 & 30 & $230 \times 10^{3}$ & 1.25 \\
$m m$ & - & $m m$ & $m m$ & $m m$ & $M P a$ & $m m$ \\
\hline
\end{tabular}

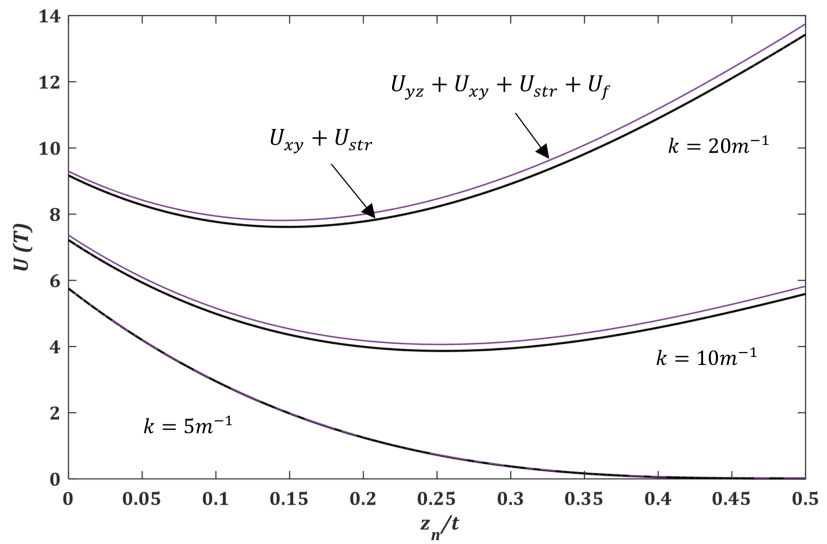

Figure 7: Variation of the strain energy with respect to $z_{n} / t$ for temperature from 273 to $373 \mathrm{~K}$

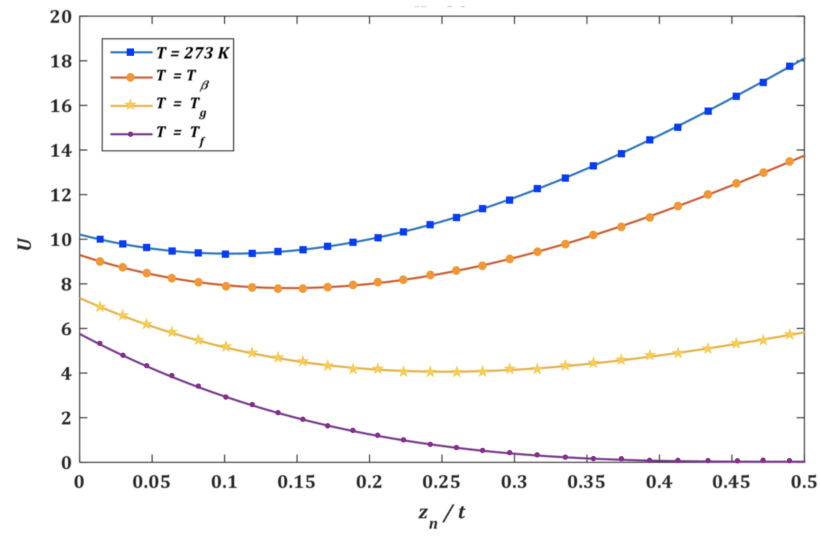

Figure 8: Strain energy of SMPC with respect to $z_{n} / t$ across the glass transition region of the SMP matrix

The influence of temperature is highlighted in Figure 8 through substitution of the properties of the temperature regulated SMP matrix from Table 1 . The curves for $\mathrm{T}=273$ $\mathrm{K}, T_{\beta}=295.2 \mathrm{~K}, T_{g}=305 \mathrm{~K}$ and $T_{f}=415.5 \mathrm{~K}$ are indicated, assuming a constant value of $k$ as $50 \mathrm{~m}^{-1}$. The total strain energy during bending of the SMPC decreased with the corresponding increase of temperature because of softening of the SMP matrix at elevated temperature. The softness of the matrix is in agreement with the change in moduli

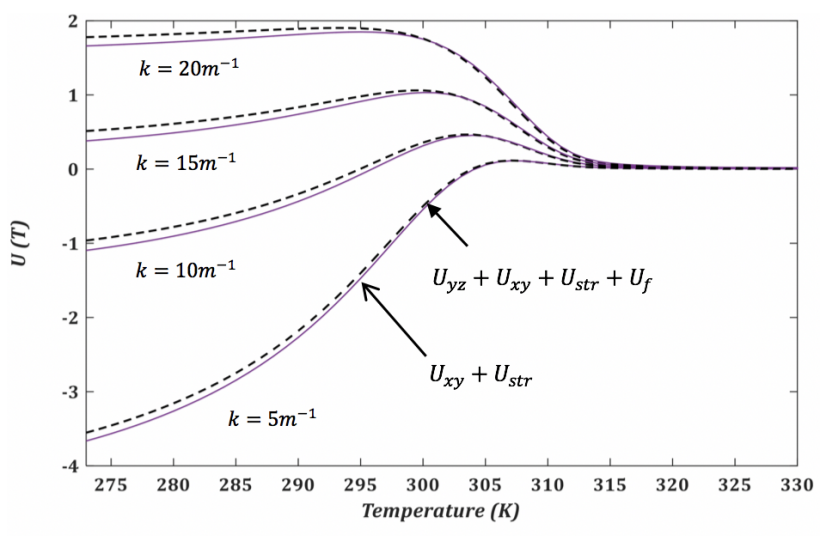

(a)

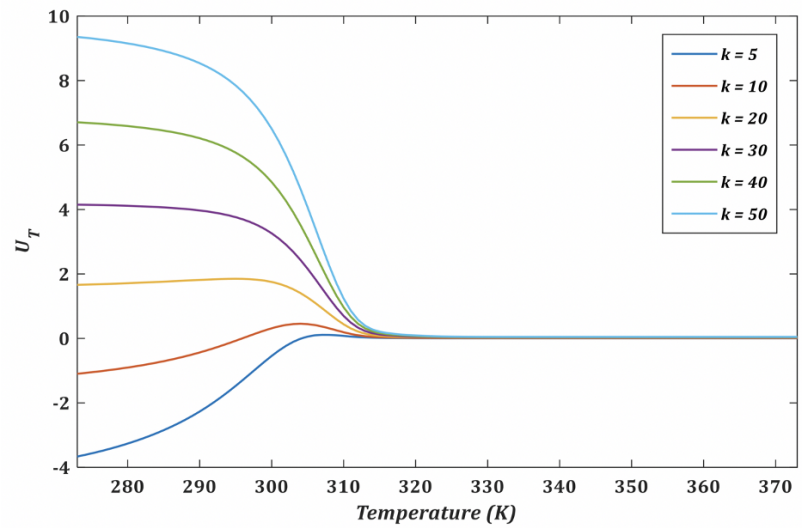

(b)

Figure 9: Variation of strain energy with respect to temperature (a) Comparison of total strain energy and sum of $U_{x y}$ and $U_{s t r}$ (b) Values of $U$ for the values of $k$ from 5 to $50 \mathrm{~m}^{-1}$

of the SMP in its glass transition region, as indicated in Figures 6(a) and 6(b).

The relationship between the total strain energy $(U)$ and the temperature variation is shown in Figure 9(a), for values of $k$ of 5, 10, 15 and $20 \mathrm{~m}^{-1}$. The total strain energy first increased significantly with respect to temperature for the values of $k$ equal to 5 and $10 \mathrm{~m}^{-1}$ and then declined slightly before becoming stable; however, for the values of 
15 and 20, initially the total strain energy increased slightly, then declined and finally followed for the same path as for $k$ equal to 5 and $10 \mathrm{~m}^{-1}$. It was also observed that that the values of $U$ were almost equal to the sums of $U_{x y}$ and $U_{s t r}$. The dynamic behavior of the strain energy is visible prior to the glass transition region, but for all the values of $k$, the energy stabilized to remain constant above $320 \mathrm{~K}$. It was also noticed that the gap between the energy curves, for each $k$, was maximum at $273 \mathrm{~K}$, which then reduced to converge at $320 \mathrm{~K}$. More results of $U$ with respect to temperature are presented for $k$ from 5 to $50 \mathrm{~m}^{-1}$ in Figure 9(b). The volatile nature of the energy is due to the sudden decrease in modulus of the SMP matrix in its glass transition region which subsequently governs the micro-buckling of the fiber in the SMPC under bending.

\subsection{Position of neutral strain plane $\left(z_{n}\right)$ and critical buckling plane $\left(z_{b}\right)$}

The placements of the neutral strain plane $\left(z_{n}\right)$ and the critical buckling plane $\left(z_{b}\right)$ is significant to locate the positions of the buckled fibers during the bending of the SMPC plate. The nature of $z_{n} / t$ and $z_{b} / t$ with respect to temperature (T) for various curvatures, $k=5,10,20$ and $50 \mathrm{~m}^{-1}$ are indicated in Figures 10 and 11. It is noticed that as the bending curvature increased, the position of $z_{n}$ and $z_{b}$ moved towards the tensile zone of the composite plate under bending. When the values of $z_{n} / t$ and $z_{b} / t$ rose above zero, then micro-buckling of the fibers in the SMPC initiated. Therefore, as the bending curvature increased, the tendency of the fibers to micro buckle increased. Moreover, due to an increase of temperature through the glass transition region, the tendency of the fibers to buckle also increased due to the decrease in moduli of the SMP matrix.

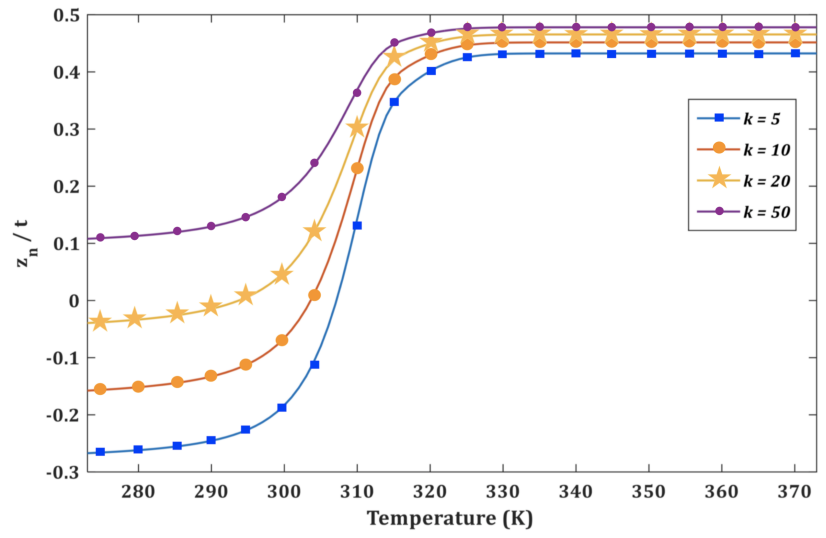

Figure 10: Variation of the position of the neutral strain surface $z_{n} / t$ with respect to temperature for various degree of bending, $k\left(m^{-1}\right)$

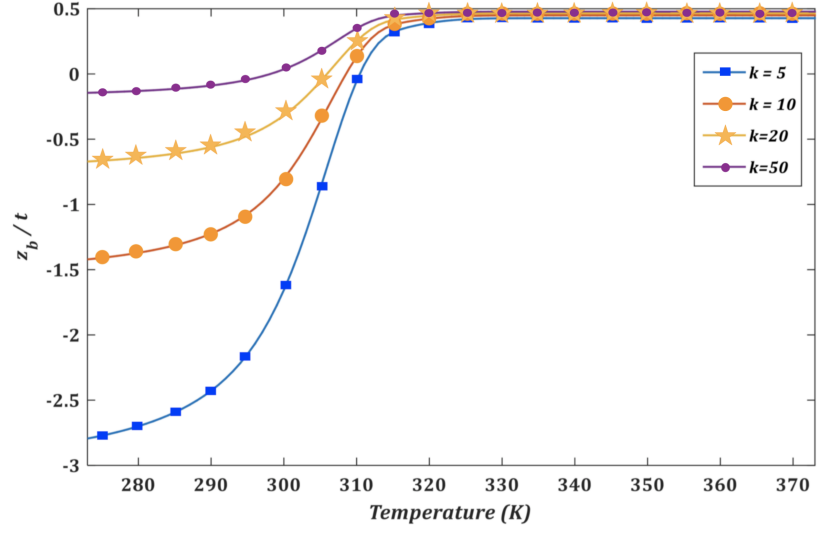

Figure 11: Variation of the position of the critical buckling surface $z_{b} / t$ with respect to temperature for various degree of bending, $k$ $\left(m^{-1}\right)$

The results obtained in this study of the position of $z_{n}$ and $z_{b}$ are in close agreement with those of Ref. [31]. Thus the trajectories of the curves in Figures 10 and 11 encompassed the entire glass transition region of the SMP matrix and presented a wholesome interpretation of microbuckling of fiber in SMPC plates under bending, which describes the phenomenon better than the one estimated only at an elevated temperature in previous works. This study also establishes the significance of temperature on the alteration of the positions of the buckling fibers in the SMPC under large bending deformations.

\subsection{Half wavelength of the fiber}

The half wavelength $(\lambda)$ of the micro buckled fiber was calculated through the simplified expression stated in Eq. (52). Two parametric studies were also performed to study the effects of volume fraction of the fibers and thickness of the SMPC.

The influence of the volume fraction of fiber reinforcement in the SMP matrix on $\lambda$ is indicated in Figure 12 for three temperatures: $T_{\beta}=295.2 \mathrm{~K}, T_{g}=305 \mathrm{~K}$ and $T_{f}=415.5$ $\mathrm{K}$. The maximum value of $\lambda$ was obtained near the volume fraction of 0.4 for all the considered temperatures. The magnitude of the wavelength increased with the rise of temperature due to the decline in modulus of the SMP. The results are in agreement with those of Ref. [30] and [31], who estimated the parameters at elevated temperatures above $T_{g}$.

The dynamic behavior of the half wavelength of the buckled fibers in the SMPC in the glass transition region of the matrix with an increase in temperature is depicted in Figure 13 for fiber volume fractions values from 20 to $70 \%$. 


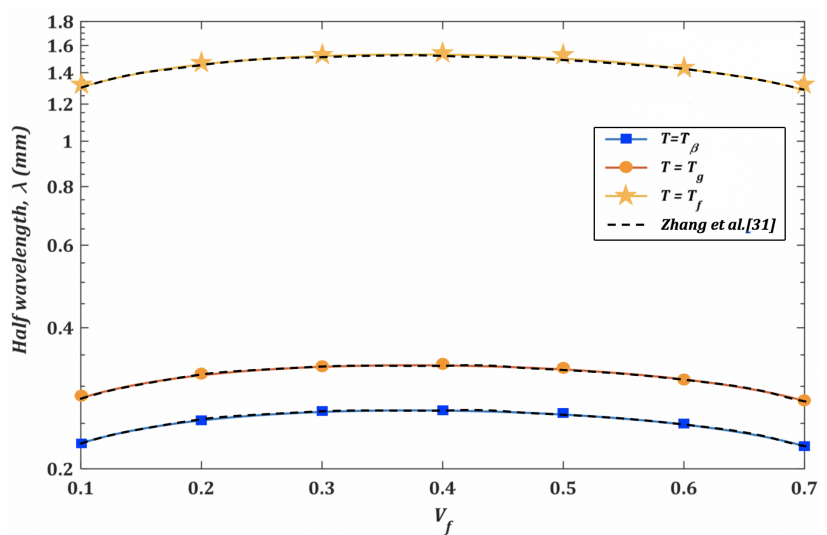

Figure 12: Variation of the half fiber buckling wavelength $(\lambda)$ with respect to fiber volume fraction at $T=T_{\beta}, T_{g}$ and $T_{f}$

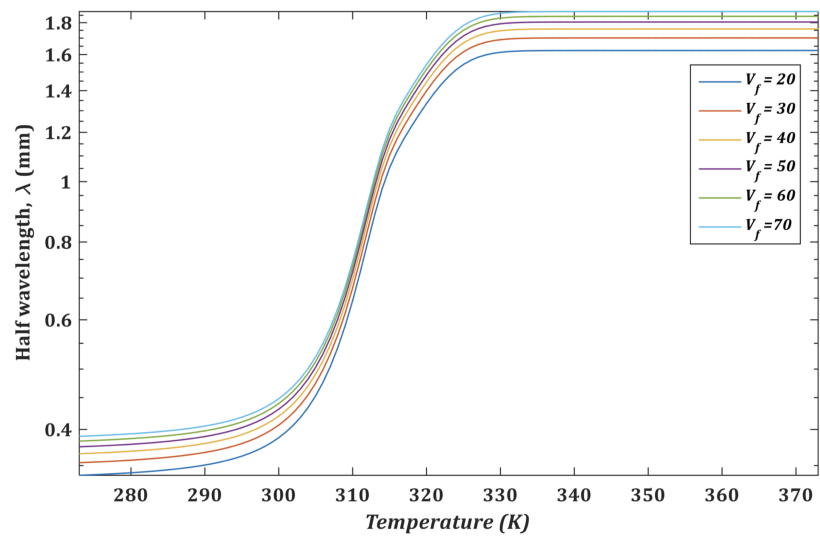

Figure 13: Variation of the half fiber buckling wavelength $(\lambda)$ with respect to temperature $(T)$ at $V_{f}$ from 20 to $70 \%$

The half wavelength was stable in the pre and post glass transition regions, with only an effect of an increase in volume fraction of the fibers being noticed, whereas within the dynamic region of the glass transition all the trajectories nearly converged. Thus, an increase of the volume fraction increased the magnitude of $\lambda$ only below and above the glass transitions region, in those regions in which the modulus of SMP material was almost constant, whereas in the glass transition region the effect of volume fraction variation was practically negligible.

The effect of thickness and temperature of the SMPC plate on the half wavelength is indicated in Figure 14, for thicknesses from $0.5 \mathrm{~mm}$ to $2.5 \mathrm{~mm}$. It was observed, that as the thickness increased, there was a consistent increase in the half wavelength for the same number of fibers which is due to the increase of gap between the adjacent fibers. The three temperatures of $T_{\beta}=295.2 \mathrm{~K}, T_{g}=305 \mathrm{~K}$ and $T_{f}=415.5$ $\mathrm{K}$ were taken into consideration and the corresponding results at elevated temperature, above $T_{g}$, were in close agreement with those of Zhang et al. [31].

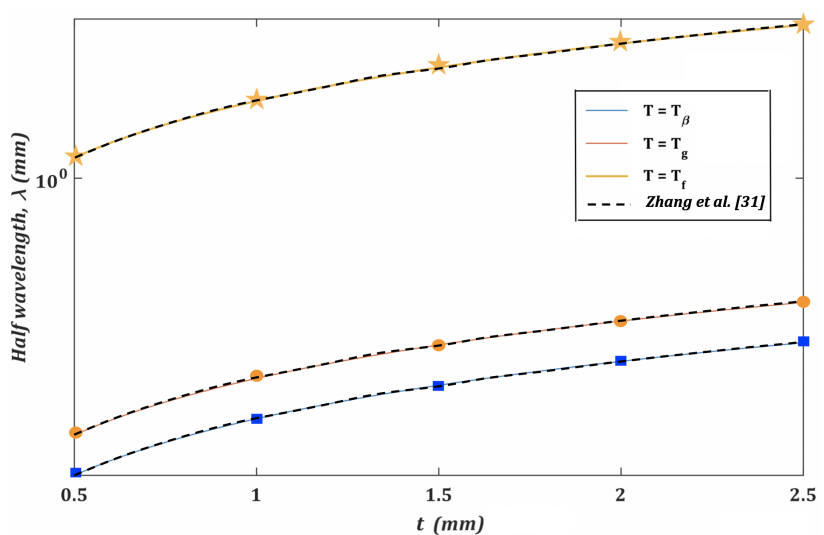

Figure 14: Variation of the half wavelength $(\lambda)$ with respect to thickness $(t)$ at $T=T_{\beta}, T_{g}$ and $T_{f}$

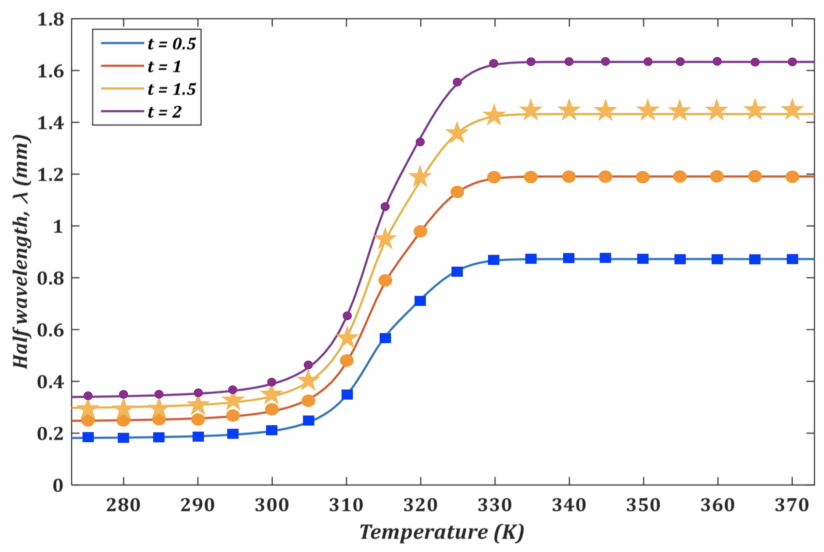

Figure 15: Variation of the half wavelength $(\lambda)$ with respect to temperature $(T)$ at $t$ from 0.5 to $2 \mathrm{~mm}$

The influence of temperature in the glass transition region of the SMP matrix on the values of $\lambda$, for the same deflection, is shown in Figure 15 for the range of thicknesses of SMPC from 0.5 to $2 \mathrm{~mm}$. It is noted that the influence of thickness on the wavelength increased at elevated temperature beyond the glass transition region of SMP as compared to it below $T_{g}$. The magnitude of $\lambda$ increased with the rise of temperature due to an enhancement in the softness of the matrix in its glass transition temperature region.

\section{Conclusions}

A comprehensive theoretical study of the micro-buckling of fibers in SMPC under large bending strain deformation is presented for the temperature range of the entire glass transition region of the SMP matrix through a minimum energy method. The fibers are considered to be 3D linearly 
elastic with the material properties of the matrix varying under the dynamic temperature conditions.

The expressions to calculate the neutral strain surface, critical buckling surface and half wavelength, as regulated by the temperature, for the SMPC plates were determined for the wide range of temperature encompassing the glass transition region of the SMP matrix. The following salient results were obtained in this study:

- The total strain energy is approximately equivalent to the sum of the strain energy in the $x y$ plane $\left(U_{x y}\right)$ and the SMPC $\left(U_{s t r}\right)$, indicating negligible values of strain energy of the fibers $\left(U_{f}\right)$ and strain energy in the $y z$ plane $\left(U_{y z}\right)$ in the SMPC.

- The magnitudes of positions of the neutral strain surface $\left(z_{n}\right)$ and critical buckling surface $\left(z_{b}\right)$ and the half wavelength $(\lambda)$ varied dynamically in the glass transition region of the SMPC.

- Variation of the volume fraction of the fibers had a negligible effect on the half wavelength in the glass transition region of the SMP matrix.

- Variations in properties of the SMP matrix regulated the nature of the micro-buckling of the fiber reinforcements, as was indicated by the dynamism of the half wavelength.

- The bending curvature had a noticeable impact on the total strain energy and positions of the neutral strain surface $\left(z_{n}\right)$ and critical buckling surface $\left(z_{b}\right)$ in the glass transition region of the SMP matrix, while it was non-effective above this region.

- The thickness had little effect on the magnitude of the micro-buckling prior to the glass transition region, whereas it had a considerable effect at elevated temperatures above $T_{g}$.

Funding information: The authors state no funding involved.

Author contributions: All authors have accepted responsibility for the entire content of this manuscript and approved its submission.

Conflict of Interests: The authors state no conflict of interest.

\section{References}

[1] Luo X, Mather PT. Conductive shape memory nanocomposites for high speed electrical actuation. Soft Mat. 2010;6(10):2146-9.
[2] Yu X, Zhou S, Zheng X, Guo T, Xiao Y, Song B. A biodegradable shape-memory nanocomposite with excellent magnetism sensitivity. Nanotechnology. 2009;20(23):235702.

[3] Li G, Wang Y, Wang S, Liu Z, Liu Z, Jiang J. A Thermo- and MoistureResponsive Zwitterionic Shape Memory Polymer for Novel SelfHealable Wound Dressing Applications. Macromol Mater Eng. 2019;304(3):1800603.

[4] Voit W, Ware T, Dasari RR, Smith P, Danz L, Simon D, et al. High-Strain Shape-Memory Polymers. Adv. Funct. Mater. 2010;20(1):162-71.

[5] Xie F, Liu L, Gong X, Huang L, Leng J, Liu, Y. Effects of Accelerated Aging on Thermal, Mechanical and Shape Memory Properties of Cyanate-Based Shape Memory Polymer: I Vacuum Ultraviolet Radiation. Polym. Degrad. Stab. 2017;138:91-7.

[6] Guo J, Wang Z, Tong L, L H, Liang W. Shape Memory and ThermoMechanical Properties of Shape Memory Polymer/Carbon Fiber Composites. Compos. Part A Appl. Sci. Manuf. 2015;76:162-171.

[7] Liu Z, Lan X, Bian W, Liu L, Li Q, Liu Y, et al. Design, Material Properties and Performances of a Smart Hinge Based on Shape Memory Polymer Composites. Compos. B. Eng. 2020;193:108056.

[8] Dao TD, Ha NS, Goo NS, Yu W. Design, Fabrication, and Bending Test of Shape Memory Polymer Composite Hinges for Space Deployable Structures. J Intell Mater Syst Struct 2018;29(8):156074.

[9] Bao M, Lou X, Zhou Q, Dong W, Yuan H, Zhang Y. Electrospun Biomimetic Fibrous Scaffold from Shape Memory Polymer of PDLLA-Co-TMC for Bone Tissue Engineering. ACS Appl Mater Interf. 2014;6(4):2611-2621.

[10] Gaj MP, Wei A, Fuentes-Hernandez C, Zhang Y, Reit R, Voit W, et al. Organic Light-Emitting Diodes on Shape Memory Polymer Substrates for Wearable Electronics. Org. Electron. 2015;25:151155.

[11] He J, Xiao G, Chen X, Qiao Y, Xu D, Lu Z. A Thermoresponsive Microfluidic System Integrating a Shape Memory Polymer-Modified Textile and a Paper-Based Colorimetric Sensor for the Detection of Glucose in Human Sweat. RSC Adv. 2019;9(41):23957-23963.

[12] Liu T, Liu L, Yu M, Li Q, Zeng C, Lan X, et al. Integrative Hinge Based on Shape Memory Polymer Composites: Material, Design, Properties and Application. Compos. Struct. 2018;206:164-176.

[13] Wang W, Yu CY, Abrego Serrano PA, Ahn S.-H. Soft Grasping Mechanisms Composed of Shape Memory Polymer Based SelfBending Units. Compos. B. Eng. 2019;164:198-204.

[14] López Jiménez F, Pellegrino S. Folding of Fiber Composites with a Hyperelastic Matrix. Int J Solids Struct 2012;49(3-4):395-407.

[15] Bishara M, Rolfes R, Allix O. Revealing Complex Aspects of Compressive Failure of Polymer Composites - Part I: Fiber Kinking at Microscale. Compos. Struct. 2017;169:105-115.

[16] Gutkin R, Pinho ST, Robinson P, Curtis PT. A Finite Fracture Mechanics Formulation to Predict Fibre Kinking and Splitting in CFRP under Combined Longitudinal Compression and In-Plane Shear. Mech. Mater. 2011;43(11):730-739.

[17] Drapier S, Grandidier JC, Potier-Ferry M. A Structural Approach of Plastic Microbuckling in Long Fibre Composites: Comparison with Theoretical and Experimental Results. Int J Solids Struct 2001;38(22-23):3877-3904.

[18] Chen Y, Liu Y, Yan Y, Zhu Y, Chen, X. Helical Coil Buckling Mechanism for a Stiff Nanowire on an Elastomeric Substrate. J Mech Phys Solids 2016;95:25-43.

[19] Li F, Scarpa F, Lan X, Liu L, Liu Y, Leng J. Bending Shape Recovery of Unidirectional Carbon Fiber Reinforced Epoxy-Based Shape 
Memory Polymer Composites. Compos. Part A Appl. Sci. Manuf. 2019;116:169-179.

[20] Rudykh S, Boyce MC. Analysis of Elasmoid Fish Imbricated Layered Scale-Tissue Systems and Their Bio-Inspired Analogues at Finite Strains and Bending. IMA J Appl Math 2014,79(5): 830-847.

[21] Rosen BW. Mechanics of Composite Strengthening. Am.Soc.Metals Seminar 1965, Chapter 3.

[22] Rudykh S, deBotton G. Instabilities of Hyperelastic Fiber Composites: Micromechanical Versus Numerical Analyses. J Elast 2012;106(2):123-147.

[23] Agoras M, Lopez-Pamies O, Ponte Castañeda P. Onset of Macroscopic Instabilities in Fiber-Reinforced Elastomers at Finite Strain. J Mech Phys Solids 2009;57(11):1828-1850.

[24] Michel JC, Lopez-Pamies O, Ponte Castañeda P, Triantafyllidis N. Microscopic and Macroscopic Instabilities in Finitely Strained Fiber-Reinforced Elastomers. J Mech Phys Solids 2010;58(11):1776-1803.

[25] Slesarenko V, Rudykh S. Microscopic and Macroscopic Instabilities in Hyperelastic Fiber Composites. J Mech Phys Solids 2017;99:471-482.

[26] Guynn EG, Ochoa 00, Bradley WL. A Parametric Study of Variables That Affect Fiber Microbuckling Initiation in Composite Laminates: Part 1-Analyses: J. Compos. Mater. 2016;26:15941616.

[27] Guynn EG, Bradley WL, Ochoa 00. A Parametric Study of Variables That Affect Fiber Microbuckling Initiation in Composite Laminates: Part 2 - Experiments. J. Compos. Mater. 1992;26:16171643.

[28] Campbell D, Maji A. Failure Mechanisms and Deployment Accuracy of Elastic-Memory Composites. J Aerosp Eng 2006;19:184193.

[29] Francis W, Lake M, Schultz M, Campbell D, Dunn M, Qi H. J. Elastic Memory Composite Microbuckling Mechanics: Closed-Form Model with Empirical Correlation. In 48th AIAA/ASME/ASCE/AHS/ASC Structures, Structural Dynamics, and Materials Conference; American Institute of Aeronautics and Astronautics.

[30] Lan X, Liu L, Liu Y, Leng J, Du S. Post Microbuckling Mechanics of Fibre-Reinforced Shape-Memory Polymers Undergoing Flexure Deformation. Mech. Mater. 2014;72:46-60.
[31] Zhang J, Dui G, Liang X. Revisiting the Micro-Buckling of Carbon Fibers in Elastic Memory Composite Plates under Pure Bending. Int. J. Mech. Sci. 2018;136:339-348.

[32] Zhang J, Dui G, Wang X. Post-Micro-Buckling of Carbon Fibers in Functionally Graded Plates under Pure Bending. Eur J Mech A Solids 2019;75:419-425.

[33] Parnes R, Chiskis A. Buckling of Nano-Fibre Reinforced Composites: A Re-Examination of Elastic Buckling. J Mech Phys Solids 2002;50:855-879.

[34] Westbrook KK, Kao PH, Castro F, Ding Y, Jerry Qi H. A 3D Finite Deformation Constitutive Model for Amorphous Shape Memory Polymers: A Multi-Branch Modeling Approach for Nonequilibrium Relaxation Processes. Mech. Mater. 2011;43:853-869.

[35] Gu J, Leng J, Sun H, Zeng H, Cai Z. Thermomechanical Constitutive Modeling of Fiber Reinforced Shape Memory Polymer Composites Based on Thermodynamics with Internal State Variables. Mech. Mater. 2019;130:9-19.

[36] Qi HJ, Nguyen TD, Castro F, Yakacki CM, Shandas R. Finite Deformation Thermo-Mechanical Behavior of Thermally Induced Shape Memory Polymers. J Mech Phys Solids 2008;56 (5):1730-1751.

[37] Tiwari N, Shaikh AA. Flexural analysis of thermally actuated fiber reinforced shape memory polymer composite. Adv Mat Res. 2019; 8(4):337-59.

[38] Cinefra M, Valvano S, Carrera E. Heat conduction and Thermal Stress Analysis of laminated composites by a variable kinematic MITC9 shell element. Curved and Layered Structures 2015;2:301320.

[39] Cinefra M, Valvano S, Carrera E. Thermal stress analysis of laminated structures by a variable kinematic MITC 9 shell element. J. Therm. Stress, 2016;39(2):121-141.

[40] Carrera E, Valvano S. A variable kinematic shell formulation applied to thermal stress of laminated structures. J. Therm. Stress, 2017;40(7):803-827.

[41] Carrera E, Valvano S. A variable ESL/LW kinematic plate formulation for free-vibration thermoelastic analysis of laminated structures. J. Therm. Stress, 2019;42(4):452-474. 